\title{
Erratum to: Hybrid maize breeding with doubled haploids: V. Selection strategies for testcross performance with variable sizes of crosses and $S_{1}$ families
}

\author{
Thilo Wegenast $\cdot$ H. Friedrich Utz •
}

C. Friedrich H. Longin - Hans Peter Maurer •

Baldev S. Dhillon · Albrecht E. Melchinger

Published online: 3 September 2010

(C) Springer-Verlag 2010

\section{Erratum to: Theor Appl Genet (2010) 120:699-708}

DOI 10.1007/s00122-009-1187-y

In the original version of the article, an error was detected in the simulation of breeding scheme $\mathrm{S}_{1}$ TC-DHTC. This error led to an overestimation of the selection gain $\left(\Delta \widehat{G}^{*}\right)$ and the probability of selecting superior genotypes $\left(\widehat{P}(q)^{*}\right)$ in this breeding scheme. The corrected results for all selection strategies of breeding scheme $\mathrm{S}_{1}$ TC-DHTC in Tables 2 and 3, as well as Fig. 1 are presented in the following pages.

The online version of the original article can be found under doi:10.1007/s00122-009-1187-y.

T. Wegenast · H. F. Utz · B. S. Dhillon · A. E. Melchinger $(\bowtie)$ Institute of Plant Breeding, Seed Science,

and Population Genetics, University of Hohenheim,

70593 Stuttgart, Germany

e-mail: melchinger@uni-hohenheim.de

T. Wegenast

e-mail: wegenast@uni-hohenheim.de

\section{F. H. Longin}

Limagrain Verneuil Holding,

BP 58 Route de Lavardac, 47600 Nérac, France

H. P. Maurer

State Plant Breeding Institute,

University of Hohenheim, 70593 Stuttgart, Germany 
Table 2 Optimum allocation of test resources maximizing the optimization criteria (OC), selection gain $\left(\Delta \widehat{G}^{*}\right)$ and the probability of selecting superior genotypes $\left(\widehat{P}(0.1 \%)^{*}\right)$, in two-stage selection with evaluation of testcross progenies of (1) DH lines at both stages (DHTC) and (2) $\mathrm{S}_{1}$ families at first stage and DH lines of $\mathrm{S}_{1}$ families at second stage $\left(\mathrm{S}_{1} \mathrm{TC}-\mathrm{DHTC}\right)$

\begin{tabular}{|c|c|c|c|c|c|c|c|}
\hline \multirow[t]{2}{*}{ Breeding scheme/selection strategy } & \multicolumn{4}{|l|}{ Optimum allocation } & \multirow[t]{2}{*}{$\mathrm{OC}$} & \multirow[t]{2}{*}{$\mathrm{SD}_{\mathrm{OC}}$} & \multirow[t]{2}{*}{$\bar{\Theta}$} \\
\hline & $N_{1}^{*} \mathrm{a}$ & $N_{2}^{*} \mathrm{~b}$ & $L_{1}^{*}$ & $L_{2}^{*}$ & & & \\
\hline \multicolumn{8}{|l|}{ Optimization criterion $\Delta \widehat{G}^{*}$} \\
\hline DHTC-1 & $5,538=3 \times 1,846$ & $392=2 \times 196$ & 2 & 14 & 3.322 & 0.385 & 0.500 \\
\hline DHTC-2a & $5,812=4 \times 1,453$ & 320 & 2 & 15 & 3.384 & 0.324 & 0.311 \\
\hline DHTC-2b & 6,152 & 330 & 2 & 12 & 3.335 & 0.340 & 0.324 \\
\hline DHTC-2c & 4,704 & 203 & 3 & 14 & 3.347 & 0.326 & 0.323 \\
\hline $\mathrm{S}_{1}$ TC-DHTC-1 & $816=4 \times 204$ & $646=2 \times 1 \times 323$ & 12 & 14 & 3.581 & 0.516 & 0.750 \\
\hline $\mathrm{S}_{1}$ TC-DHTC-2a & $800=5 \times 160$ & $660=3 \times 1 \times 220$ & 12 & 14 & 3.610 & 0.485 & 0.663 \\
\hline $\mathrm{S}_{1} \mathrm{TC}-\mathrm{DHTC}-2 \mathrm{~b}$ & $824=4 \times 206$ & 753 & 10 & 14 & 3.624 & 0.484 & 0.581 \\
\hline $\mathrm{S}_{1} \mathrm{TC}-\mathrm{DHTC}-2 \mathrm{c}$ & $725=5 \times 145$ & 781 & 11 & 14 & 3.612 & 0.494 & 0.584 \\
\hline \multicolumn{8}{|l|}{ Optimization criterion $\widehat{P}(0.1 \%)^{*}$} \\
\hline DHTC-1 & $5,655=3 \times 1,885$ & $400=2 \times 200$ & 2 & 13 & 0.631 & 0.295 & 0.500 \\
\hline DHTC-2a & $5,644=4 \times 1,411$ & 348 & 2 & 15 & 0.671 & 0.252 & 0.316 \\
\hline DHTC-2b & 6,204 & 320 & 2 & 12 & 0.640 & 0.258 & 0.322 \\
\hline DHTC-2c & 4,640 & 220 & 3 & 14 & 0.651 & 0.258 & 0.322 \\
\hline $\mathrm{S}_{1} \mathrm{TC}-\mathrm{DHTC}-1$ & $820=4 \times 205$ & $680=2 \times 1 \times 340$ & 13 & 12 & 0.771 & 0.295 & 0.750 \\
\hline $\mathrm{S}_{1}$ TC-DHTC-2a & $775=5 \times 155$ & $735=3 \times 1 \times 245$ & 11 & 14 & 0.773 & 0.265 & 0.566 \\
\hline $\mathrm{S}_{1} \mathrm{TC}-\mathrm{DHTC}-2 \mathrm{~b}$ & $1,057=7 \times 151$ & 712 & 9 & 13 & 0.779 & 0.258 & 0.541 \\
\hline $\mathrm{S}_{1}$ TC-DHTC-2c & $828=6 \times 138$ & 804 & 11 & 12 & 0.775 & 0.262 & 0.576 \\
\hline
\end{tabular}

Assumptions: a budget of 20,000 testcross plot equivalents, variance component ratios VC2, and a correlation $\rho_{P}=0.71$ between the mean performance of the parental lines and the mean genotypic value of the testcross performance of their progeny

$N_{j}^{*}=$ optimum number of test candidates in stage $j, L_{j}^{*}=$ optimum number of test locations in stage $j, S D=$ the standard deviation, and $\bar{\Theta}=$ the average coefficient of coancestry among the selected DH lines

${ }^{\mathrm{a}}$ DHTC -1 and 2a: number of crosses $\times$ DH lines within crosses, DHTC $-2 \mathrm{~b}$ and $2 \mathrm{c}$ : the number of DH lines within crosses depended on the rank of the cross; $S_{1}$ TC-DHTC: number of crosses $\times \mathrm{S}_{1}$ families within crosses

${ }^{\mathrm{b}}$ DHTC-1: number of crosses $\times$ DH lines within crosses, DHTC-2: number of DH lines; $S_{1}$ TC-DHTC -1 and 2 a: number of crosses $\times \mathrm{S}_{1}$ families within crosses $\times$ DH lines within $S_{1}$ families; $S_{1}$ TC-DHTC-2b and 2c: the number of $S_{1}$ families within crosses and DH lines within $S_{1}$ families depended on the rank of the cross and the $S_{1}$ family

Table 3 Optimum allocation of test resources maximizing the optimization criteria $(\mathrm{OC})$, selection gain $\left(\Delta \widehat{G}^{*}\right)$ and the probability of selecting superior genotypes $\left(\widehat{P}(0.1 \%)^{*}\right)$, in two-stage selection with evaluation of testcross progenies of (1) DH lines at both stages (DHTC-2a) and (2) $S_{1}$ families at first stage and DH lines of $S_{1}$ families at second stage $\left(\mathrm{S}_{1}\right.$ TC-DHTC-2c) and its dependence on the phenotypic correlation $\rho_{P}$ (between the mean performance of the parents and the mean genotypic value of the testcross performance of their progenies), the variance component ratios (VC), and the budget in terms of testcross plot equivalents

\begin{tabular}{|c|c|c|c|c|c|c|c|c|c|c|}
\hline \multirow[t]{2}{*}{ Breeding scheme/selection strategy } & \multicolumn{3}{|c|}{ Assumptions } & \multicolumn{4}{|l|}{ Optimum allocation } & \multirow[t]{2}{*}{$\mathrm{OC}$} & \multirow[t]{2}{*}{$\mathrm{SD}_{\mathrm{OC}}$} & \multirow[t]{2}{*}{$\bar{\Theta}$} \\
\hline & Budget & $\mathrm{VC}$ & $\rho_{P}$ & $N_{1}^{*}$ a & $N_{2}^{*}$ & $L_{1}^{*}$ & $L_{2}^{*}$ & & & \\
\hline \multicolumn{11}{|l|}{ Optimization criterion $\Delta \widehat{G}^{*}$} \\
\hline DHTC-2a & 10,000 & 2 & 0.71 & $2,874=3 \times 958$ & 191 & 2 & 13 & 3.257 & 0.316 & 0.340 \\
\hline DHTC-2a & 20,000 & 2 & 0.71 & $5,812=4 \times 1,453$ & 320 & 2 & 15 & 3.384 & 0.324 & 0.311 \\
\hline DHTC-2a & 40,000 & 2 & 0.71 & $9,325=5 \times 1,865$ & 427 & 3 & 14 & 3.491 & 0.322 & 0.296 \\
\hline DHTC-2a & 20,000 & 1 & 0.71 & $9,556=4 \times 2,389$ & 390 & 1 & 13 & 3.662 & 0.309 & 0.318 \\
\hline DHTC-2a & 20,000 & 3 & 0.71 & $3,564=2 \times 1,782$ & 232 & 4 & 14 & 3.089 & 0.317 & 0.399 \\
\hline DHTC-2a & 20,000 & 2 & 0.50 & $6,069=7 \times 867$ & 297 & 2 & 14 & 3.070 & 0.369 & 0.268 \\
\hline
\end{tabular}


Table 3 continued

\begin{tabular}{|c|c|c|c|c|c|c|c|c|c|c|}
\hline \multirow[t]{2}{*}{ Breeding scheme/selection strategy } & \multicolumn{3}{|c|}{ Assumptions } & \multicolumn{4}{|l|}{ Optimum allocation } & \multirow[t]{2}{*}{$\mathrm{OC}$} & \multirow[t]{2}{*}{$\mathrm{SD}_{\mathrm{OC}}$} & \multirow[t]{2}{*}{$\bar{\Theta}$} \\
\hline & Budget & $\mathrm{VC}$ & $\rho_{P}$ & $N_{1}^{*} \mathrm{a}$ & $N_{2}^{*}$ & $L_{1}^{*}$ & $L_{2}^{*}$ & & & \\
\hline $\mathrm{S}_{1}$ TC-DHTC-2c & 10,000 & 2 & 0.71 & $267=3 \times 89$ & 686 & 9 & 10 & 3.434 & 0.505 & 0.592 \\
\hline $\mathrm{S}_{1}$ TC-DHTC-2c & 20,000 & 2 & 0.71 & $725=5 \times 145$ & 781 & 11 & 14 & 3.639 & 0.494 & 0.584 \\
\hline $\mathrm{S}_{1}$ TC-DHTC-2c & 40,000 & 2 & 0.71 & $848=4 \times 212$ & 2,391 & 10 & 12 & 3.751 & 0.484 & 0.561 \\
\hline $\mathrm{S}_{1}$ TC-DHTC-2c & 20,000 & 1 & 0.71 & $888=4 \times 222$ & 980 & 10 & 11 & 3.991 & 0.498 & 0.601 \\
\hline $\mathrm{S}_{1}$ TC-DHTC-2c & 20,000 & 3 & 0.71 & $522=3 \times 174$ & 856 & 13 & 14 & 3.224 & 0.476 & 0.605 \\
\hline $\mathrm{S}_{1} \mathrm{TC}-\mathrm{DHTC}-2 \mathrm{c}$ & 20,000 & 2 & 0.50 & $794=12 \times 66$ & 720 & 11 & 14 & 3.376 & 0.524 & 0.551 \\
\hline \multicolumn{11}{|l|}{ Optimization criterion (OC) $\widehat{P}(0.1 \%)^{*}$} \\
\hline DHTC-2a & 10,000 & 2 & 0.71 & $3,153=3 \times 1,051$ & 149 & 2 & 12 & 0.595 & 0.273 & 0.338 \\
\hline DHTC-2a & 20,000 & 2 & 0.71 & $5,644=4 \times 1,411$ & 348 & 2 & 15 & 0.671 & 0.252 & 0.316 \\
\hline DHTC-2a & 40,000 & 2 & 0.71 & $9,375=5 \times 1,875$ & 447 & 3 & 13 & 0.730 & 0.225 & 0.296 \\
\hline DHTC-2a & 20,000 & 1 & 0.71 & $9,728=4 \times 2,432$ & 371 & 1 & 13 & 0.833 & 0.197 & 0.315 \\
\hline DHTC-2a & 20,000 & 3 & 0.71 & $3,222=2 \times 1,611$ & 342 & 4 & 14 & 0.500 & 0.284 & 0.397 \\
\hline DHTC-2a & 20,000 & 2 & 0.50 & $6,216=7 \times 888$ & 293 & 2 & 13 & 0.479 & 0.271 & 0.271 \\
\hline $\mathrm{S}_{1} \mathrm{TC}-\mathrm{DHTC}-2 \mathrm{c}$ & 10,000 & 2 & 0.71 & $267=3 \times 89$ & 686 & 9 & 10 & 0.683 & 0.321 & 0.566 \\
\hline $\mathrm{S}_{1}$ TC-DHTC-2c & 20,000 & 2 & 0.71 & $775=5 \times 155$ & 735 & 11 & 14 & 0.778 & 0.265 & 0.566 \\
\hline $\mathrm{S}_{1}$ TC-DHTC-2c & 40,000 & 2 & 0.71 & $852=4 \times 213$ & 2,388 & 10 & 12 & 0.829 & 0.242 & 0.559 \\
\hline $\mathrm{S}_{1}$ TC-DHTC-2c & 20,000 & 1 & 0.71 & $1,115=5 \times 223$ & 868 & 9 & 10 & 0.924 & 0.152 & 0.599 \\
\hline $\mathrm{S}_{1}$ TC-DHTC-2c & 20,000 & 3 & 0.71 & $477=3 \times 159$ & 896 & 13 & 14 & 0.570 & 0.321 & 0.610 \\
\hline $\mathrm{S}_{1}$ TC-DHTC-2c & 20,000 & 2 & 0.50 & $737=11 \times 67$ & 820 & 11 & 13 & 0.648 & 0.316 & 0.552 \\
\hline
\end{tabular}

$N_{j}^{*}=$ optimum number of test candidates in stage $j, L_{j}^{*}=$ optimum number of test locations in stage $j, S D=$ the standard deviation, and $\bar{\Theta}=$ the average coefficient of coancestry among the selected DH lines

${ }^{a}$ DHTC-2a: number of crosses $\times$ DH lines within crosses; $S_{1}$ TC-DHTC-2c: number of crosses $\times S_{1}$ families within crosses

Fig. 1 Selection gain $(\Delta \widehat{G})$ and the probability of selecting superior genotypes $(\widehat{P}(0.1 \%))$ as a function of the number of crosses in the first stage $\left(N_{1_{C}}\right)$ for selection strategies 1 (open square), $2 \mathrm{a}$ (open circle), $2 \mathrm{~b}$ (open triangle), and 2c (open diamond) in breeding scheme DHTC (solid symbols) and $\mathrm{S}_{1} \mathrm{TC}-\mathrm{DHTC}$ (hollow symbols)
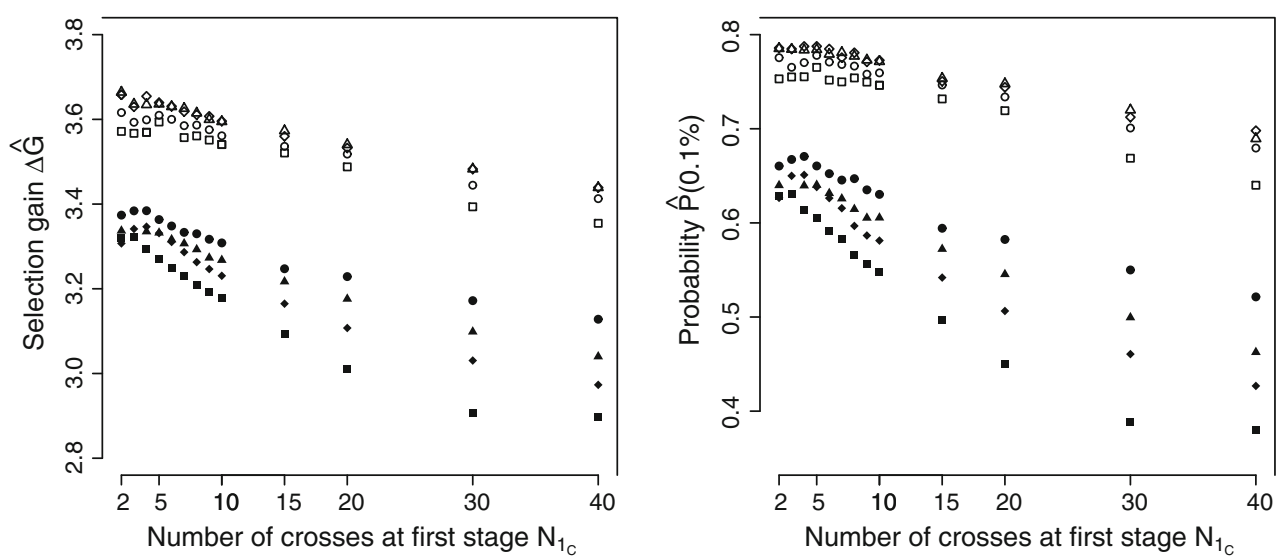